\title{
Plasmoid formation for multiple onset substorms: observations of the Japanese Lunar Mission "Kaguya"
}

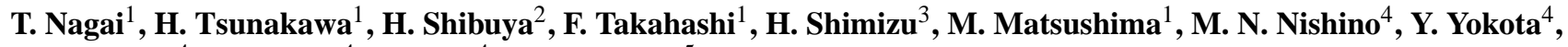 \\ K. Asamura ${ }^{4}$, T. Tanaka ${ }^{4}$, Y. Saito ${ }^{4}$, and O. Amm ${ }^{5}$ \\ ${ }^{1}$ Tokyo Institute of Technology, Tokyo, Japan \\ ${ }^{2}$ Kumamoto University, Kumamoto, Japan \\ ${ }^{3}$ University of Tokyo, Tokyo, Japan \\ ${ }^{4}$ Institute of Space and Astronautical Science, Japan Aerospace Exploration Agency, Japan \\ ${ }^{5}$ Finnish Meteorological Institute, Helsinki, Finland
}

Received: 1 September 2008 - Revised: 3 December 2008 - Accepted: 3 December 2008 - Published: 6 January 2009

\begin{abstract}
The Japanese Lunar Mission "Kaguya" carried out its first magnetic field and plasma measurements in the Earth's magnetotail on 22 December 2007. Fortuitously, three well-defined multiple onset substoms took place. Kaguya was located in the premidnight magnetotail at radial distances of $56 R_{E}$ and observed plasmoids and/or traveling compression regions (TCRs). Although the present study is based on limited data sets, important issues on multiple onset substorms can be examined. Each onset in a series of onsets releases a plasmoid, and magnetic reconnection likely proceeds to tail lobe field lines for each onset. Since the duration of each plasmoid is less than $5 \mathrm{~min}$, these observations imply that magnetic reconnection for each onset can develop fully to the tail lobe field lines and be quenched within this timescale.
\end{abstract}

Keywords. Magnetospheric physics (Magnetotail; Storms and substorms) - Space plasma physics (Magnetic reconnection)

\section{Introduction}

The Japanese Lunar Mission "Kaguya" was launched on 14 September 2007. The Kaguya spacecraft encircles the moon at an altitude of $100 \mathrm{~km}$ with an orbital period of $118 \mathrm{~min}$. Its first plasma and magnetic field observations in the Earth's magnetotail were carried out on 22 December 2007. Kaguya traveled near the northern tail lobe/plasma sheet boundary from $\left(-52,+21,+1 R_{E} \mathrm{GSM}\right)$ at 12:00 UT to

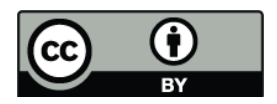

Correspondence to: $\mathrm{T}$. Nagai

(nagai@geo.titech.ac.jp) $\left(-55,+13,+7 R_{E}\right.$ GSM) at 24:00 UT on 22 December 2007. Fortuitously, three multiple onset substorms took place and Kaguya observed plasmoids and traveling compression regions (TCRs) during these substorms.

Characteristics of plasmoids and TCRs have already been studied extensively (e.g. Hones et al., 1984; Slavin et al., 1984, 1989, 1993, 1998, 2002, 2003; Baker et al., 1987; Moldwin and Hughes, 1992a, b, 1993; Nagai et al., 1994; Ieda et al., 1998). Since formation of a plasmoid is thought to be a natural consequence of magnetic reconnection in the near-Earth magnetotail (e.g. Hones et al., 1984), we can investigate development of magnetic reconnection during multiple onset substorms. In this paper we address two questions. One is whether or not each onset in a multiple onset substorm creates a plasmoid. Plasmoids and TCRs are often observed successively during substorm activity. Slavin et al. (1992) examined ISEE 3 observations at radial distances of 76-80 $R_{E}$ during an extended interval of substorm activity and found that all of the substorms produced one or more plasmoids and/or TCRs. However, one-to-one correspondence between each onset and a plasmoid is not fully examined. The other is whether or not tail lobe open field lines are reconnected in the magnetic reconnection process for each onset. This question does not seem to be explored properly.

\section{Observations}

Figure 1 presents the ground magnetic field data (the $\mathrm{H}$ component and $d H / d t$ ) at the mid-latitude station Kakioka (geomagnetic latitude is $27.5^{\circ}$ and magnetic local midnight is 14:45 UT) for the period of 13:00-14:00 UT on 22 December 2007. There are four Pi2 bursts starting at 13:24, 13:29,

Published by Copernicus Publications on behalf of the European Geosciences Union. 


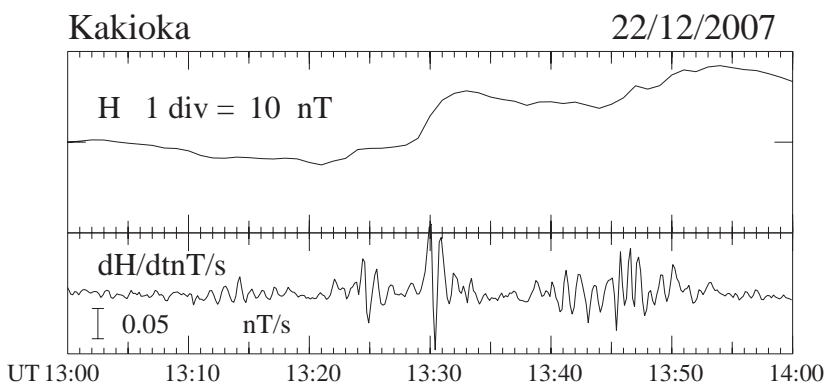

Fig. 1. Ground magnetic field data at Kakioka (the $\mathrm{H}$ component and $d H / d t$ for Pi2) for the period 13:00-14:00 UT on 22 December 2007.

13:40, and 13:45 UT. A mid-latitude positive bay starts with the first Pi2 burst. The second and fourth Pi2 bursts are accompanied with clear positive changes in the $\mathrm{H}$ component of the magnetic field. The data from another mid-latitude station Urumqi (magnetic latitude is $34.2^{\circ}$ and magnetic local midnight is 18:00 UT) also show these four Pi2 bursts at the same times. Furthermore, the $\mathrm{D}$ variation is positive at Urumqi and negative at Kakioka, respectively, so that the central meridian of this substorm activity is located between these two stations. The geosynchronous spacecraft LANL97A is located just west of the Kakioka meridian (local midnight is 14:05 UT), so that the location of this spacecraft is the best for monitoring the substorm activity. Electron injections are observed at 13:25, 13:32, 13:46, and 13:50 UT, and electron and proton fluxes do not show any variations for the period 12:00-13:25 UT (not shown). Hence, this substorm is identified as a multiple onset substorm with a sequence of four onsets (e.g. McPherron, 1991; Pytte et al., 1976a, b, Nagai et al., 1983), hereafter, called the first multiple onset substorm.

Figure 2 presents the magnetic field data from Kaguya for the period of 13:20-14:00 UT. The magnetometer on Kaguya is described by Shimizu et al. (2008). At 13:25 and 13:31 UT, two plasmoids are identified in the magnetic field data. The second plasmoid is a typical one, which has a bipolar (norththen-south) variation in $B_{z}$. During a depression in $B_{x}, B_{y}$ becomes largely positive. The first plasmoid is rather atypical. There is a small bipolar variation in $B_{z}$ initially, however, a main body of this plasmoid has a largely positive variation in $B_{z}$. Furthermore, a largely positive deflection in $B_{y}$ is observed inside this plasmoid. The spacecraft Geotail observes the interplanetary magnetic field (IMF) in the front of the magnetosphere at $\left(29.0,0.0,-7.0 R_{E}\right.$ GSM). There is no large IMF $B_{y}$ (less than $3 \mathrm{nT}$ ) in this interval (not shown). Kaguya is located near the $125^{\circ} \mathrm{E}$ longitude meridian in the Southern Hemisphere of the moon, and there is no strong magnetic anomaly in the moon's surface in this region. These atypical magnetic field behaviors are frequently observed for plasmoids in the Kaguya observations (for the period through August 2008). It is possible that they are

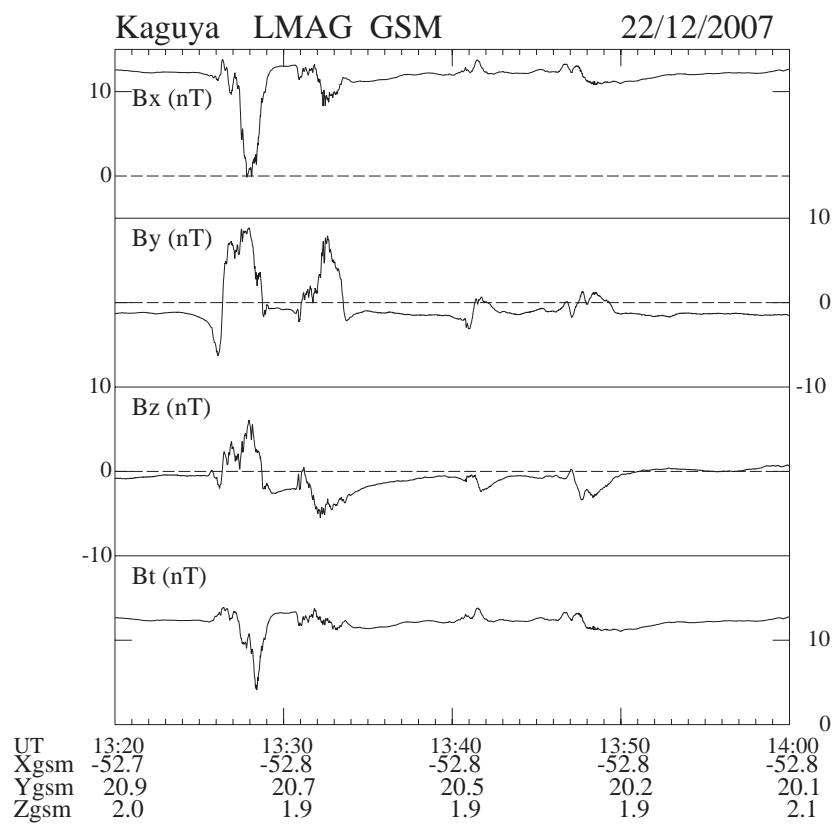

Fig. 2. Kaguya magnetic field observations of a series of plasmoids and TCRs recorded for the period 13:20-14:00 UT on 22 December 2007.

caused by the existence of the moon, however, origin of the atypical variations in the magnetic field is not identified at present. Two TCRs are identified in the magnetic field data at 13:41 and 13:46 UT. Hence, there are four plasmoid signatures which are well associated with the four onsets recorded on the ground and at synchronous orbit.

Figure 3 presents the plasma data from Kaguya as energytime diagrams for the period of 13:20-13:36 UT. The plasma instrument is described by Saito et al. (2008). Threedimensional distribution functions from 32 energy channels are obtained with a time resolution of $16 \mathrm{~s}$ for electrons and $32 \mathrm{~s}$ for ions for this time interval. For each energy channel, angular distributions for ions and electrons can be examined (a half hemisphere is divided into $16 \times 4$ for ions and $64 \times 16$ for electrons). The ion detector (IEA) was operated in the solar wind mode in this time interval, so that ion counts were extremely low. The ion mass analyzer (IMA) with less angular resolution was also operated. Tailward flowing ions with energies of $>2 \mathrm{keV}$ are observed for the period of 13:26:04-13:29:16 UT. Lower-energy $(<1 \mathrm{keV})$ ions are simultaneously detected in the ion mass analyzer (not shown). Tail lobe electrons are observed until 13:25:48 UT. Electrons with energies of higher than $0.5 \mathrm{keV}$ appear at 13:25:48 UT and then electrons with energies of higher than $1 \mathrm{keV}$ appear at 13:26:04 UT. These energetic electrons are observed until 13:29:00 UT. This electron behavior is consistent with the plasmoid signature in the magnetic field data. Plasmas constituting the plasma sheet and the plasma sheet/tail lobe boundary are easily identified in ion and electron data from 


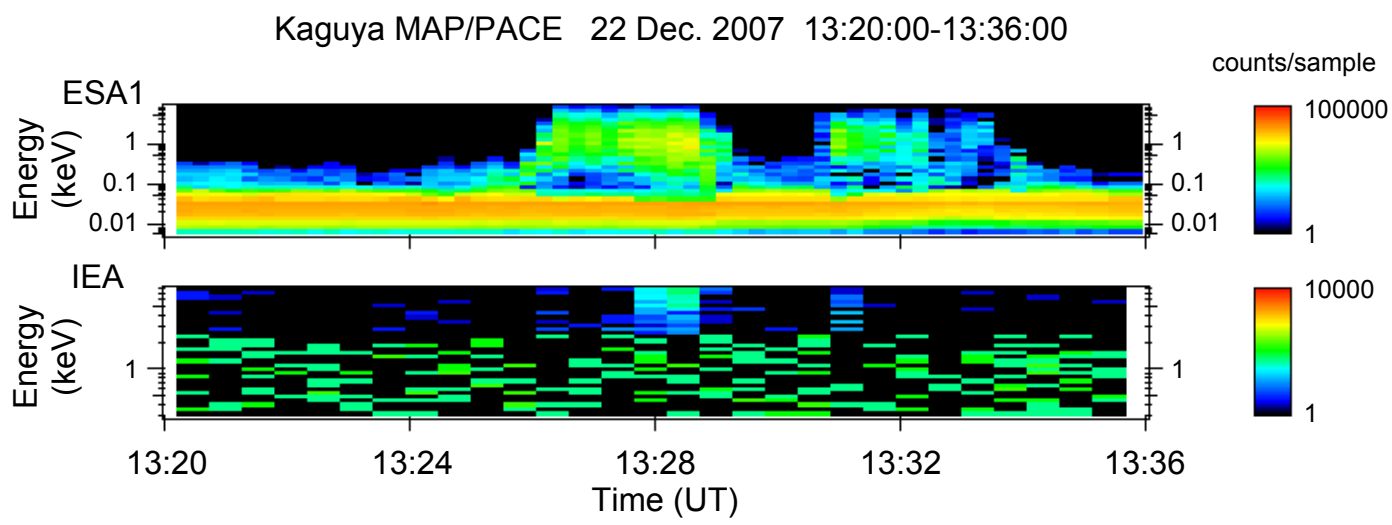

Fig. 3. Kaguya electron (the top panel) and ion (the bottom panel) measurements for the period 13:20-13:36 UT on 22 December 2007. Ion observations were made in a mode that were less sensitive to ions with energies $<2.5 \mathrm{keV}$.

this plasma instrument. However, plasmas indicating the normal plasma sheet and its boundary region are not identified with detailed examinations of the distribution functions for this interval. Hence, the plasma observations strongly indicate that Kaguya directly enters the plasmoid from the tail lobe. For the second plasmoid, energetic electrons appear at 13:31:52 UT, when the magnetic field data show an initial signature of the plasmoid. This also indicates that Kaguya directly enters the plasmoid from the tail lobe.

Figure 4 shows the magnetic field data from Kaguya for the second multiple onset substorm. Three Pi2 bursts are observed at 15:39, 15:45, and 15:49 UT at Kakioka and Urumqi (not shown). Unfortunately, there are no particle observations near midnight at synchronous orbit and only one gradual increase in the electron flux is observed at LANL97A (near 02:00 MLT). A plasmoid signature is observed at 15:39 UT, while two TCRs are observed at 15:45 and 15:49 UT. Energetic electrons appear at 15:39:08 UT (not shown). This plasmoid is another example that Kaguya directly enters the plasmoid from the tail lobe.

Figure 5 shows the magnetic field data from Kaguya for the third multiple onset substorm. For this substorm, the magnetic field data from the IMAGE magnetometer network in Scandinavia are examined. There are at least five intensifications of the westward electrojet at 19:25, 19:32, 19:46, 19:58, and 20:10 UT. The spacecraft LANL-02A (local midnight is 19:30 UT) observes electron injections at 19:25, 19:35, 19:48, 20:05, and 20:18 UT. Five TCR-like events at 19:28, 19:34, 19:48, 20:01, and 20:12 UT are identified in the Kaguya magnetic field data. The magnetic signatures of the first and second events (at 19:28 and 19:34 UT) are not typical TCRs, and these are accompanied with appearances of high-energy $(>1 \mathrm{keV})$ electrons. These seem to be classified as boundary layer plasmoids (Moldwin and Hughes, 1992b). The third, fourth, and fifth events are identified as TCRs.

In summary, the observations by Kaguya on 22 December 2007 indicate that there is one-to-one correspondence between each onset in a multiple onset substorm and a plasmoid. Furthermore, the plasma and magnetic field data show that the outer boundary of the plasmoid touches the tail lobe.

\section{Discussion and conclusions}

During the three multiple onset substorms on 22 December 2007, Kaguya is located at $\mathrm{X}=-52$ to $-55 R_{E}$ and observes a plasmoid signature for each onset. The results from the Geotail observations show that magnetic reconnection in association with a substorm onset usually takes place in the premidnight plasma sheet at $\mathrm{X}=-20$ to $-30 R_{E}$ (e.g. Nagai et al., 1998a, 2005). It is difficult to station spacecraft continuously in the plasma sheet or near the plasma sheet/tail lobe boundary in the distant tail, because of large-amplitude motion of the distant tail. The moon is thought to be one of the best stations for monitoring plasmoids. Indeed, Kaguya did not miss any plasmoid that was released on this day. For the first substorm, the time delays relative to the Pi2 onsets are 2, 2, 1, and $1 \mathrm{~min}$. For the second substorm, the time delays relative to the Pi2 onsets are $-1,1$, and $0 \mathrm{~min}$. The average time delay is $1 \mathrm{~min}$. If we assume that magnetic reconnection takes place at $\mathrm{X}=-25 R_{E}$ and its signals travel earthward and tailward at the same speed, the signal speed is $530 \mathrm{~km} \mathrm{~s}^{-1}$. This speed is consistent with the plasma flow speed estimated by the ion mass analyzer data, and is a representative speed of the plasmoid observed by Geotail in this distance range (Ieda et al., 1998). For the third substorm, time delays are 3, 2, 2, 3 , and $2 \mathrm{~min}$. Since these time delays are those relative to the intensifications of the westward electrojet in the IMAGE network data, they might become larger. It is noted that there is no systematic change among the time delays in the sequence of events. It is hard to examine whether magnetic reconnection takes place at the same position in the near-Earth tail or it successively moves tailward (or Earthward) in a sequence of onsets. For the first and third substorms, the final two electron injections are delayed to the ground onset (and the 


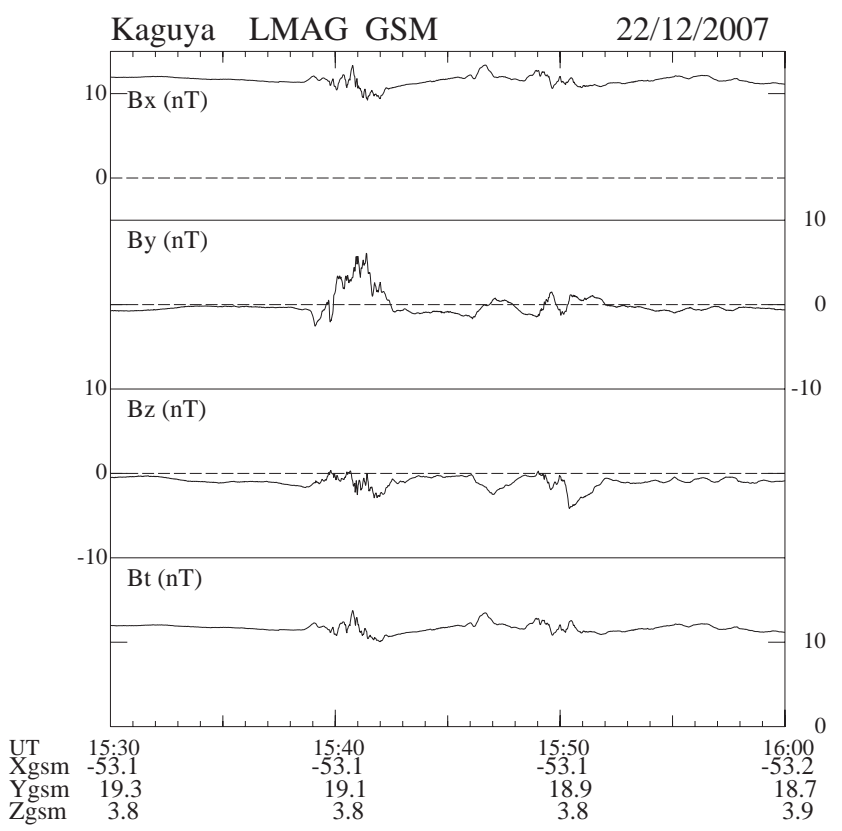

Fig. 4. Kaguya magnetic field observations of a series of plasmoids and/or TCRs recorded for the period 15:30-16:00 UT on 22 December 2007.

plasmoid arrival) by $4-5 \mathrm{~min}$. This probably indicates that these particle injections at synchronous orbit take place far from the midnight meridian. This is already reported by Nagai et al. (1983) and is consistent with the idea that the onset sector moves westward (e.g. Wiens and Rostoker, 1975).

The present study clearly shows that each onset in a multiple onset substorm releases a plasmoid. If we assume that plasmoid speed is $500 \mathrm{~km} \mathrm{~s}^{-1}$ and its duration is $2 \mathrm{~min}$, the size of a plasmoid is approximately $10 R_{E}$ at $\mathrm{X}=-50 R_{E}$. Hence, a plasmoid created at each onset can develop well and its size is comparable to the average plasmoid size at this radial distance (Ieda et al., 1998). The Kaguya observations provide evidence that the spacecraft directly enters a plasmoid from the tail lobe, at least, for the first plasmoid in the series of plasmoids. Since the outer envelope of a plasmoid extends to the tail lobe, it is reasonably interpreted that magnetic reconnection proceeds to tail lobe field lines. It is interesting to discuss the present results to the previous observations in the near-Earth magnetotail by Geotail. Nagai et al. (1998a) reported three tailward plasma flow bursts with negative $B_{z}$, each of which is associated with a $\mathrm{Pi} 2$ burst, when Geotail continuously stayed inside the plasma sheet. Hence, this is an example of plasma flow observations in the near-Earth magnetotail for multiple onset substorms. The plasma sheet became stagnant in the intervals between a pair of Pi2 bursts. Probably, a multiple onset substorm is not caused by any modulation of the reconnection rate in a single magnetic reconnection process. It is likely that magnetic reconnection starts and ceases at several times at the same

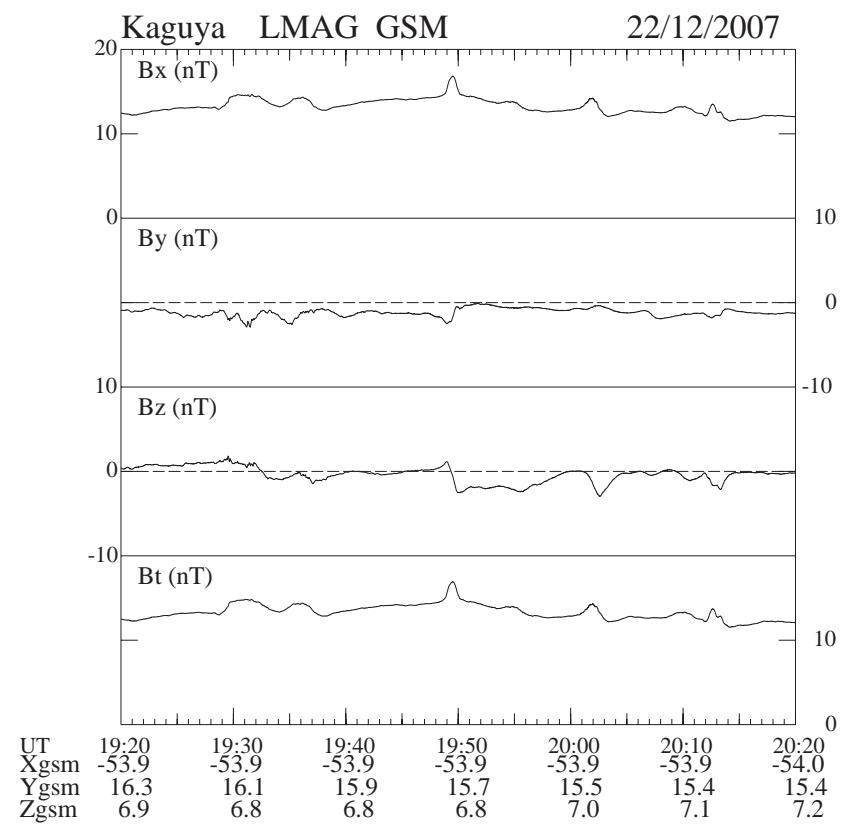

Fig. 5. Kaguya magnetic field observations of a series of TCRs recorded for the period 19:20-20:20 UT on 22 December 2007.

position or different sites for a multiple onset substorm and that each magnetic reconnection process can develop fully even for a short duration (the average time duration of the plasmoids is $3.5 \mathrm{~min}$ for the three multiple onset substorms). Nagai et al. (1998b) examined magnetic reconnection in the near-Earth plasma sheet for a pseudobreakup. Fast tailward plasma flows with negative $B_{z}$ are embedded in the plasma sheet and the outer plasma sheet is stationary, so that magnetic reconnection likely involves only closed plasma sheet flux tubes for a pseudobreakup (see also Ohtani et al., 2004). Whether or not open tail lobe flux tubes are reconnected seems to control development of substorm processes.

The present observations provide two important results on the development of magnetic reconnection in the near-Earth magnetotail during a multiple onset substorm. Each onset in a series of onsets produces a plasmoid, so that each onset likely corresponds to one magnetic reconnection process. In each event, magnetic reconnection likely proceeds to tail lobe field lines. Since the duration of one plasmoid observation is less than $5 \mathrm{~min}$ and the interval of two successive plasmoids can be less than $5 \mathrm{~min}$, magnetic reconnection proceeds quickly and all plasma sheet field lines can be reconnected in less than $5 \mathrm{~min}$. In the present observations, socalled post-plasmoid plasma sheet signatures are not evident. In the distant tail (at $220 R_{E}$ ), tailward flows, which are a manifestation of the post-plasmoid plasma sheet, continue for more than $40 \mathrm{~min}$ (e.g. Baker et al., 1987). Magnetic reconnection in the near-Earth tail seems to be quenched rather quickly during multiple onset substorms. The plasma and magnetic field observations in the magnetotail by Kaguya 
continue and the data are available. Atypical magnetic field signatures are frequently observed for plasmoids. Plasmoid signatures are observed when Kaguya stays in the plasma sheet, the plasma sheet/tail lobe boundary, and the tail lobe. The ion and electron signatures presented in this study are repeatedly observed. The results here are initial ones based on the limited data sets, however, the observations on 22 December 2007 can be judged to be representative. More comprehensive analyses will be needed using the data for the whole Kaguya operation period.

Acknowledgements. The authors thank the members of the Kaguya/PACE team. The ground magnetic field data were supplied by Kyoto University World Data Center. The Los Alamos spacecraft data were examined at http://leadbelly.lanl.gov. The Geotail magnetic field data are available at http://www.darts.isas.jaxa.jp/stp/ cef/cef.cgi.

Topical Editor R. Nakamura thanks M. Moldwin and another anonymous referee for their help in evaluating this paper.

\section{References}

Baker, D. N., Anderson, R. C., Zwickl, R. D., and Slavin, J. A.: Average plasma and magnetic field variations in the distant magnetotail associated with near-Earth substorm effects, J. Geophys. Res., 71-81, 1987.

Hones Jr., E. W., Baker, D. N., Bame, S. J., Feldman, W. C., Gosling, J. T., McComas, D. J., Zwickl, R. D., Slavin, J. A., Smith, E. J., and Tsurutani, B. T.: Structure of the magnetotail at $220 R_{E}$ and its response to geomagnetic activity, Geophys. Res. Lett., 11, 5-7, 1984.

Ieda, A., Machida, S., Mukai, T., Saito, Y., Yamamoto, T., Nishida, A., Terasawa, T., and Kokubun, S.: Statistical analysis of the plasmoid evolution with Geotail observations, J. Geophys. Res., 103, 4453-4465, 1998.

McPherron, R. L.: Physical processes producing magnetospheric substorms and magnetic storms, in: Geomagnetism, vol. 4, edited by: Jacobs, J. A., 593-739, Academic Press, London, 1991.

Moldwin, M. B. and Hughes, W. J.: On the formation and evolution of plasmoids: A survey of ISEE 3 geotail data, J. Geophys. Res., 97, 19259-19282, 1992a.

Moldwin, M. B. and Hughes, W. J.: Plasmoid observations in the distant plasma sheet boundary layer, Geophys. Res. Lett., 19, 1911-1914, 1992b.

Moldwin, M. B. and Hughes, W. J.: Geomagnetic substorm association of plasmoids, J. Geophys. Res., 98, 81-88, 1993.

Nagai, T., Baker, D. N., and Higbie, P. R.: Development of substorm activity in multiple-onset substorms at synchronous orbit, J. Geophys. Res., 88, 6994-7004, 1983.

Nagai, T., Takahashi, K., Kawano, H., Yamamoto, T., Kokubun, S., and Nishida, A.: Initial GEOTAIL survey of magnetic substorm signatures in the magnetotail, Geophys. Res. Lett., 21(25), 29912994, 1994.

Nagai, T., Fujimoto, M., Saito, Y., Machida, S., Terasawa, T., Nakamura, R., Yamamoto, T., Mukai, T., Nishida, A., and Kokubun, S.: Structure and dynamics of magnetic reconnection for sub- storm onsets with Geotail observations, J. Geophys. Res., 103(3), 4419-4440, 1998a.

Nagai, T., Fujimoto, M., Nakamura, R., Saito, Y., Mukai, T., Yamamoto, T., Nishida, A., Kokubun, S., Reeves, G. D., and Lepping, R. P.: Geotail observations of a fast tailward flow at $X_{\mathrm{GSM}}=-15 R_{E}$, J. Geophys. Res., 103, 23543-23550, $1998 \mathrm{~b}$.

Nagai, T., Fujimoto, M., Nakamura, R., Baumjohann, W., Ieda, A., Shinohara, I., Machida, S., Saito, Y., and Mukai, T.: Solar wind control of the radial distance of the magnetic reconnection site in the magnetotail, J. Geophys. Res., 110, A09208, doi:10.1029/2005JA011207, 2005.

Ohtani, S., Ueno, G., Yamaguchi, R., Singer, H., Creutzberg, F., Yumoto, K., Kitamura, K., and Mukai, T.: Tail dynamics during the growth phase of the November 24, 1996, substorm event: Near-Earth reconnection confined in the plasma sheet, J. Geophys. Res., 109, A05211, doi:10.1029/2003JA010299, 2004.

Pytte, T., McPherron, R. L., and Kokubun, S.: The ground signatures of the expansion phase during multiple onset substorms, Planet. Space Sci., 24, 1115-1132, 1976a.

Pytte, T., McPherron, R. L., Kivelson, M. G., West Jr., H. I., and Hones Jr., E. W.: Multiple-satellite studies of magnetospheric substorms: Radial dynamics of the plasma sheet, J. Geophys. Res., 81, 5921-5933, 1976 b.

Saito, Y., Yokota, S., Asamura, K., Tanaka, T., Akiba, R., Fujimoto, M., Hasegawa, H., Hayakawa, H., Hirahara, M., Hoshino, M., Machida, S., Mukai, T., Nagai, T., Nagatsuma, T., Nakamura, M., Oyama, K.-I., Sagawa, E., Sasaki, S., Seki, K., and Terasawa, T.: Low-energy charged particle measurement by MAP-PACE onboard SELENE, Earth, Planets Space, 60, 375-385, 2008.

Shimizu, H., Takahashi, F., Horii, N., Matsuoka, A., Matsushima, M., Shibuya, H., and Tsunakawa, H.: Ground calibration of the high-sensitivity SELENE lunar magnetometer LMAG, Earth Planets Space, 60, 353-363, 2008.

Slavin, J. A., Smith, E. J., Tsurutani, B. T., Sibeck, D. G., Singer, H. J., Baker, D. N., Gosling, J. T., Hones, E. W., and Scarf, F. L.: Substorm associated traveling compression regions in the distant tail: ISEE-3 geotail observations, Geophys. Res. Lett., 11, 657660, 1984.

Slavin, J. A., Baker, D. N., Craven, J. D., Elphic, R. C., Fairfield, D. H., Frank, L. A., Galvin, A. B., Hughes, W. J., Manka, R. H., Mitchell, D. G., Richardson, I. G., Sanderson, T. R., Sibeck, D. J., Smith, E. J., and Zwickl, R. D.: CDAW 8 observations of plasmoid signatures in the geomagnetic tail: An assessment, J. Geophys. Res., 94, 15153-15175, 1989.

Slavin, J. A., Smith, M. F., Mazur, E. L., Baker, D. N., Iyemori, T., Singer, H. J., and Greenstadt, E. W.: ISEE 3 plasmoid and TCR observations during an extended interval of substorm activity, Geophys. Res. Lett., 19, 825-828, 1992.

Slavin, J. A., Smith, M. F., Mazur, E. L., Baker, D. N., Hones Jr., E. W., Iyemori, T., and Geenstadt, E. W.: ISEE 3 observations of traveling compression regions in the Earth's magnetotail, J. Geophys. Res., 98, 15425-15446, 1993.

Slavin, J. A., Fairfield, D. H., Kuznetsova, M. M., Owen, C. J., Lepping, R. P., Taguchi, S., Mukai, T., Saito, Y., Yamamoto, T., Kokubun, S., Lui, A. T. Y., and Reeves, G. D.: ISTP observations of plasmoid ejection: IMP 8 and Geotail. J. Geophys. Res. 103, 119-133, 1998.

Slavin, J. A., Fairfield, D. H., Lepping, R. P., Hesse, M., Ieda, A., Tanskanen, E., Ostgaard, N., Mukai, T., Nagai, T., Singer, H. J., 
and Sutcliffe, P. R.: Simultaneous observations of earthward flow bursts and plasmoid ejection during magnetospheric substorms, J. Geophys. Res., 107(A7), 1015, doi:10.1029/2000JA003501, 2002.

Slavin, J. A., Lepping, R. P., Gjerloev, J., Fairfield, D. H., Hesse, M., Owen, C. J., Moldwin, M. B., Nagai, T., Ieda, A., and Mukai, T.: Geotail observations of magnetic flux ropes in the plasma sheet, J. Geophys. Res., 108(A1), 1015, doi:10.1029/2002JA009557, 2003.
Wiens, R. G. and Rostoker, G.: Characteristics of the development of the westward electrojet during the expansion phase of magnetospheric substorms, J. Geophys. Res., 80, 2109-2128, 1975. 